\title{
A generalized Kerker condition for highly directive nanoantennas
}

\author{
R. Alaee ${ }^{* 1}$, R. Filter ${ }^{2}$, D. Lehr ${ }^{3}$, F. Lederer ${ }^{2}$, C. Rockstuhl ${ }^{1,4}$ \\ ${ }^{1}$ Institute of Theoretical Solid State Physics, Karlsruhe Institute of Technology, \\ Wolfgang-Gaede-Str. 1, 76128 Karlsruhe, Germany \\ ${ }^{2}$ Institute of Condensed Matter Theory and Solid State Optics, Abbe Center of Photonics \\ Friedrich-Schiller-University Jena, Max-Wien-Platz 1, 07743 Jena, Germany \\ ${ }^{3}$ Institute of Applied Physics, Abbe Center of Photonics \\ Friedrich-Schiller-University Jena, Max-Wien-Platz 1, 07743 Jena, Germany \\ ${ }^{4}$ Institute of Nanotechnology, Karlsruhe Institute of Technology, P.O. Box 3640, 76021 Karlsruhe, Germany \\ *Corresponding author: rasoul.khanghah@kit.edu
}

Compiled June 15, 2015

A nanoantenna with balanced electric and magnetic dipole moments, known as the first Kerker condition, exhibits a directive radiation pattern with zero backscattering. In principle, a nanoantenna can provide even better directionality if higher order moments are properly balanced. Here, we study a generalized Kerker condition at the example of a nanoring nanontenna supporting electric dipole and electric quadrupole moments. Nanoring antennas are well suited since both multipole moments can be almost independently tuned to meet the generalized Kerker condition. (C) 2015 Optical Society of America

OCIS codes: $240.6680,250.5403010 .1350290 .2558290 .5825$

Engineering the scattering of optical nanoantennas is a progressing field [1-4]. This is promoted by the ability of nanoantennas to support multiple resonances dominated by different electromagnetic multipole moments, i.e. beyond the electric dipole moment [5-7]. Varying size, shape, and material of nanoantennas causes intriguing scattering effects that rely on the interference of these resonances such as superscattering $[7,8]$, cloaking $[9,10]$, a control of the polarization state of radiation [11], or enhanced directionality in the scattering response [12-19]. Especially the latter function is extremely exciting considering application perspectives, for which an optical wireless nanoantenna link is a prominent example [20].

To achieve a directional pattern with zero backscattered field, Kerker suggested to exploit the interference of the electric and magnetic dipole modes of a scatterer [21]. Recently, the so-called Kerker condition has attracted considerable attention both theoretically and experimentally in order to realize directional emission from dielectric and metallic nanoantennas [13-17,22-25].

But nanoantennas can be tailored to not just sustain resonances dominated by dipolar moments, but higher order multipole moments can be sustained as well. It must be possible to systematically exploit them for similar purposes.

Here, we present a fundamental approach to design directive nanoantennas with zero backscattering based on a generalization of the Kerker condition that considers electric quadrupole moments as well. Various nanoantennas have been investigated that exhibit such higher order multipole moments to achieve a directional response $[12,25-27]$. We study the application of the generalized Kerker condition to a plasmonic nanoring antenna. Nanoring antennas [Fig. 1 (a)] have been explored because of their promising applications, e.g. for nonlin-
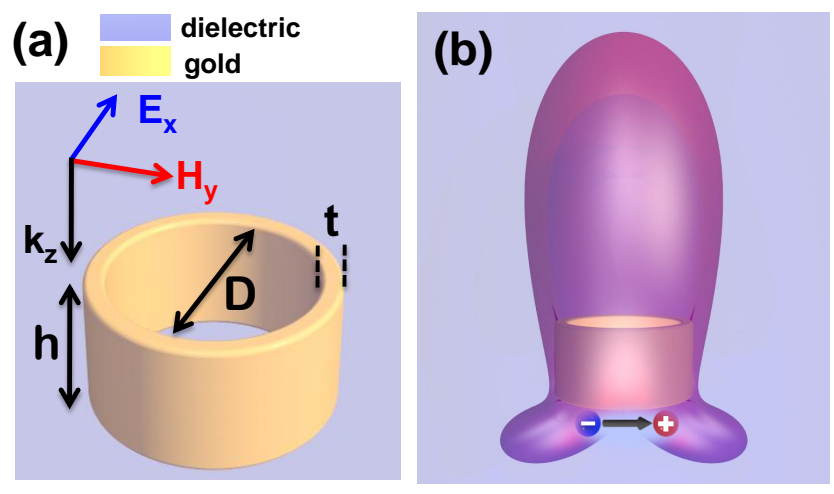

Fig. 1. (a) Schematic of the considered nanoantenna that is embedded in a homogenous host medium. (b) Artistic view of the radiation pattern of the nanoantenna.

ear effects on the nanoscale and in quantum plasmonics [28-34]. We show that for specific parameters plasmonic nanorings can support both an electric dipole and quadrupole mode. The geometry can be tuned to meet the generalized Kerker condition, i.e. both moments are balanced. Meeting the generalized Kerker condition suppresses the nanoring's backward scattering and enhances its forward scattering [Fig. 1 (b)]. We study its directional scattering upon plane wave illumination but also the directional far-field emission of a dipole emitter placed close to the nanoring. Consequences of meeting the generalized Kerker condition on the Purcell factor and the antenna efficiency are also discussed.

We start by considering the radiated far-field of a nanoantenna with an induced electric dipole moment $p_{\mathrm{x}}$, a magnetic dipole moment $m_{\mathrm{y}}$, and an electric 


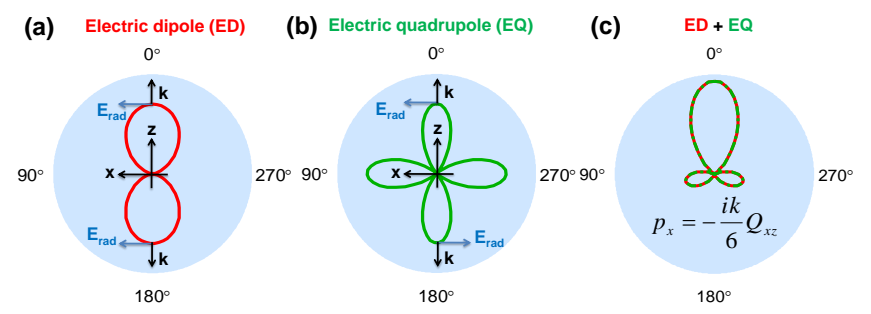

Fig. 2. Radiation pattern for different multipole moments in the $x z$-plane. (a) Electric dipole moment $\left(p_{\mathrm{x}}\right)$, i.e. $\left|\mathbf{E}_{\mathrm{far}}\right|^{2} \propto \cos ^{2} \theta$. The blue arrows indicate the phase of the radiated field. (b) Electric quadrupole moment $\left(Q_{\mathrm{xz}}\right)$, i.e. $\left.\left|\mathbf{E}_{\mathrm{far}}\right|^{2} \propto \cos ^{2} 2 \theta\right)$. (c) Superposition of electric dipole and quadrupole moments, i.e. $\left|\mathbf{E}_{\mathrm{far}}\right|^{2} \propto(\cos \theta+$ $\cos 2 \theta)^{2}$, when the generalized Kerker condition is fulfilled [cf. Eq. (3) with $\left.m_{\mathrm{y}}=0\right]$, i.e. $p_{\mathrm{x}}=-i \frac{k}{6} Q_{\mathrm{xz}}$.

quadrupole moment $Q_{\mathrm{xz}}$. It reads as [35]

$$
\begin{aligned}
\mathbf{E}_{\mathrm{far}}(\mathbf{r})= & \frac{k^{2}}{4 \pi \epsilon} p_{\mathrm{x}} \frac{e^{i k r}}{r}(-\sin \varphi \hat{\varphi}+\cos \theta \cos \varphi \hat{\theta}) \\
& -\frac{Z k^{2}}{4 \pi} m_{\mathrm{y}} \frac{e^{i k r}}{r}(\cos \theta \sin \varphi \hat{\varphi}-\cos \varphi \hat{\theta}) \\
& -\frac{i k^{3}}{24 \pi \epsilon} Q_{\mathrm{xz}} \frac{e^{i k r}}{r}(-\sin \varphi \cos \theta \hat{\varphi}+\cos 2 \theta \cos \varphi \hat{\theta})
\end{aligned}
$$

where $r, \theta$, and $\phi$ are the spherical coordinates. The host medium permittivity is $\varepsilon_{\mathrm{r}}, k=\sqrt{\varepsilon_{r}} \omega / c$ is the wavenumber for an angular frequency $\omega, Z=\sqrt{\frac{\mu_{0}}{\varepsilon_{0} \epsilon_{r}}}$ is the impedance of host, and $c$ the speed of light. Using Eq. (1), the backward radar scattering cross section of the nanoantenna can be defined as

$$
\begin{aligned}
\sigma_{\text {Backward }} & =\lim _{r \rightarrow \infty} 4 \pi r^{2} \frac{\left|\mathbf{E}_{\mathrm{far}}(\varphi=0, \theta=\pi)\right|^{2}}{\left|\mathbf{E}_{\mathrm{inc}}\right|^{2}} \\
& =\frac{k^{4}}{4 \pi \epsilon^{2}\left|\mathbf{E}_{\mathrm{inc}}\right|^{2}}\left|p_{\mathrm{x}}-\frac{\sqrt{\epsilon_{r}} m_{\mathrm{y}}}{c}+\frac{i k}{6} Q_{\mathrm{xz}}\right|^{2}
\end{aligned}
$$

where $\left|\mathbf{E}_{\text {inc }}\right|$ is the modulus of the incident electric field. According to Eq. (2), zero backscattering occurs if the so-called generalized Kerker condition

$$
p_{\mathrm{x}}-\frac{\sqrt{\varepsilon_{r}} m_{\mathrm{y}}}{c}+\frac{i k}{6} Q_{\mathrm{xz}}=0
$$

is fulfilled. Equation (3) reduces to the well-known Kerker condition $p_{\mathrm{x}}-\frac{\sqrt{\varepsilon_{r}} m_{\mathrm{y}}}{c}=0$ [21], for a vanishing quadrupole moment. To take additional higher order multipole moments into account, Eq. (3) can be easily extended. Furthermore, the forward radar scattering cross section of the nanoantenna reads as

$$
\begin{aligned}
\sigma_{\text {Forward }} & =\lim _{r \rightarrow \infty} 4 \pi r^{2} \frac{\left|\mathbf{E}_{\mathrm{far}}(\varphi=0, \theta=0)\right|^{2}}{\left|\mathbf{E}_{\mathrm{inc}}\right|^{2}} \\
& =\frac{k^{4}}{4 \pi \varepsilon^{2}\left|\mathbf{E}_{\mathrm{inc}}\right|^{2}}\left|p_{\mathrm{x}}+\frac{\sqrt{\varepsilon_{r}} m_{\mathrm{y}}}{c}-\frac{i k}{6} Q_{\mathrm{xz}}\right|^{2}
\end{aligned}
$$
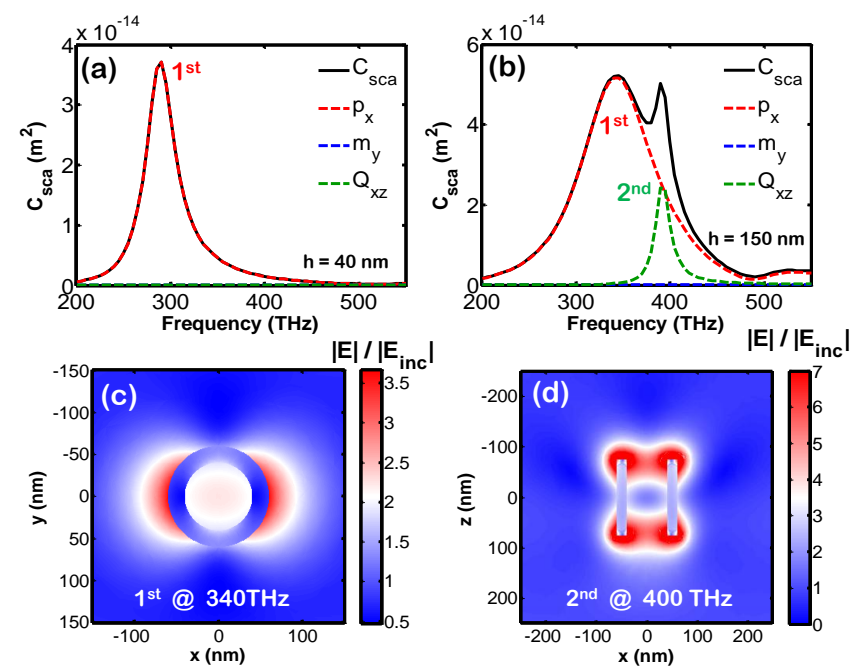

Fig. 3. Plane wave excitation of nanorings. Frequency dependent total scattering cross sections and contributions from different multipole moments for nanorings with two different heights, $h=40 \mathrm{~nm}[(\mathrm{a})]$ and $h=150 \mathrm{~nm}$ (1) [(b)]; electric dipole moment $p_{\mathrm{x}}$ (red dashed line), magnetic dipole moment $m_{\mathrm{y}}$ (blue dashed line), and electric quadrupole moment $Q_{\mathrm{xz}}$ (green dashed line). (c) and (d) Field distributions $\left(E_{\mathrm{x}}\right)$ of the nanoring with $h=150 \mathrm{~nm}$ for the first $[(\mathrm{c})]$ and second mode $[(\mathrm{d})]$.

Hence, if Eq. (3) is fulfilled, a constructive interference in forward direction is achieved in addition to the destructive one in backward direction.

To illustrate the physical mechanism behind the generalized Kerker condition, the radiation pattern of both an electric dipole $p_{\mathrm{x}}$ and an electric quadrupole moment $Q_{\mathrm{xz}}$ in the $x z$-plane $\left(\varphi=0^{\circ}\right)$ are shown in Fig. 2 (a)(b). Here, we consider $m_{\mathrm{y}}=0$, since the nanoring investigated later exhibits a negligible magnetic response. Then, if Eq. (3) holds, the electric field radiated by an electric dipole and electric quadrupole interfere constructively at $\theta=0^{\circ}$ [Fig. 2 (c)], i.e. the radiated fields are in phase in forward direction [cf. Fig. 2 (a)-(b)]. On the other hand, there is no backscattering $\left(\theta=180^{\circ}\right)$ because of the destructive interference in this direction.

Equation (3) provides a general guideline to design nanoantennas without backscattering and enhanced forward scattering on the base of multipolar interference. To demonstrate its applicability, the scattering response of a plasmonic nanoring will be investigated now. A schematic of this nanoring is depicted in Fig. 1. We assumed that the thickness of the gold layer is $t=20 \mathrm{~nm}$ with a height of $h=40-150 \mathrm{~nm}$. The diameter of the inner nanoring is $D=80 \mathrm{~nm}$. The nanoring is embedded in a homogenous host medium and illuminated by an $x$-polarized plane wave [Fig. 1 (a)].

Figures 3 (a) and (b) show the scattering cross sections of two nanorings of different heights $(h=40,150 \mathrm{~nm})$. The contribution of all multipole moments are calculated by the multipole analysis of the scattered field [36]. 

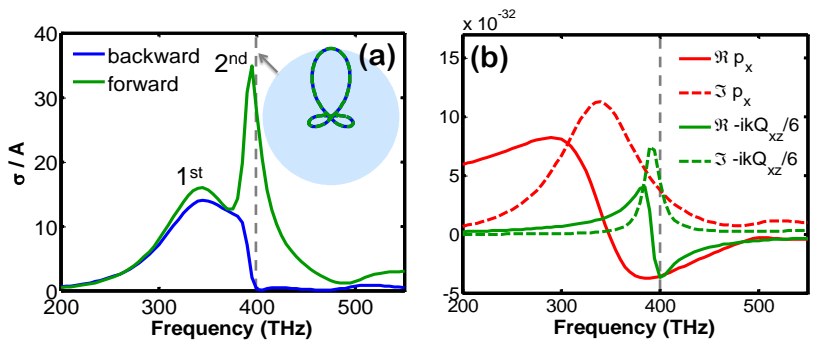

Fig. 4. Plane wave excitation of the nanoring with $h=150 \mathrm{~nm}$, radiation pattern. (a) Normalized forward (green line) and backward (blue line) scattering cross sections. Inset: The far-field pattern at $\nu_{2}=400 \mathrm{THz}$, which is identical to the theoretically predicted one [Fig. 2 (c)]. (b) The real and imaginary parts of the electric dipole moment $\left(p_{x}\right)$ and the rescaled electric quadrupole moment $\left(-i \frac{k}{6} Q_{\mathrm{xz}}\right)$. Obviously, Eq. (3) holds for both imaginary and real parts at $\nu_{2}$ (grey dashed line).

For the $40 \mathrm{~nm}$ nanoring the scattering response can be fully described by an electric dipole resonance around $\nu \approx 290 \mathrm{THz}$ [Fig. 3 (a)]. This nanoring scatters in forward and backward direction identically. By increasing the height of the nanoring to $h=150 \mathrm{~nm}$, the dipole resonance blueshifts to $\nu_{1} \approx 340 \mathrm{THz}$, but, most importantly, a second mode emerges around $\nu_{2} \approx 400 \mathrm{THz}$ [Fig. 3 (b)]. This mode can be explained by a Fabry-Perot oscillation of surface plasmons that bounce between the top and bottom of the nanoring [37,38]. According to the multipole expansion [36], the new mode is an electric quadrupole (green dashed line). Nevertheless, the first mode has a strong contribution to the scattering cross section $C_{\text {sca }}$ at $\nu_{2}$ as well. In fact, $C_{\text {sca }}$ is enhanced at $\nu_{2}$ due to the superposition of the two modes, which is known as superscattering $[7,8]$. This effect can be maximized by proper overlapping the different modes of the nanoring. Note that the contribution of magnetic dipoles is negligible in the entire frequency domain.

From now we will only consider the nanoring with $h=150 \mathrm{~nm}$ because of its interesting scattering features, i.e. directive pattern. The field distributions of the first and second mode for the nanoring at selected cross-sections are depicted in Fig. 3 (c) and (d), respectively. For the first mode $\left(\nu_{1} \approx 340 \mathrm{THz}\right)$, the electric field inside the nanoring is almost uniformly distributed $[28,39,40]$. However, the second mode exhibits a strong field enhancement around the upper and lower terminations of the nanoring. Furthermore, the field varies strongly inside the nanoring. Figure 4 (a) shows the forward and backward radar scattering cross sections for the investigated nanoring [Eqs. (2) and (4)]. Both are similar below $330 \mathrm{THz}$ due to the dominating dipolar response. However, above $400 \mathrm{THz}$, the forward scattering is significantly enhanced while the backward scattering is considerably suppressed.

So far we claimed that the generalized Kerker condition [Eq. (3)] explains the suppression of the backward
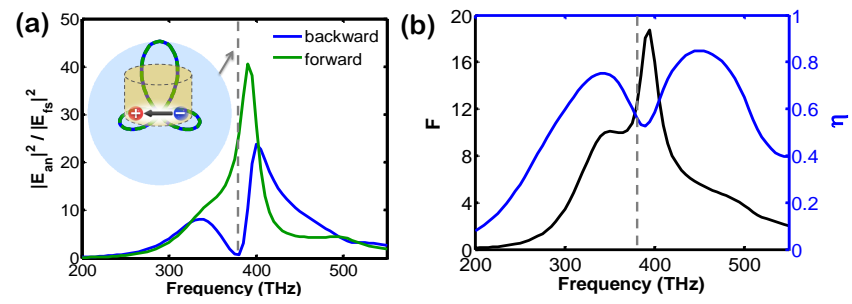

Fig. 5. Electric dipole excitation. (a) Normalized forward and backward far-fields: $E_{\mathrm{fs}}$ is the radiated electric field by a point electric dipole without nanoantenna and $E_{\text {an }}$ the same in the presence of the nanoring. Inset: Highly directive radiation pattern at $\nu_{\text {dip }} \approx 380 \mathrm{THz}$. (b) Purcell factor $F$ (black line) and antenna efficiency $\eta$ (blue line).

scattering and enhancement of the forward scattering due to the coherent interference of the involved multipoles. To confirm this claim, the imaginary and real parts of the electric dipole and quadrupole moments of the nanoring are sketched in Fig. 4 (b). It can be seen that Eq. (3) $\left[p_{\mathrm{x}}=-i \frac{k}{6} Q_{\mathrm{xz}}\right.$ for $\left.m_{\mathrm{y}}=0\right]$ holds for $\nu=\nu_{2}$. Hence, the Kerker condition explains the scattering properties, since they agree perfectly with the theoretical predictions [ $c f$. inset of Fig. 4 (a) and Fig. 2 (c)].

A direct transfer of the results from far-field excitations to near-field ones, e.g. by a close-by dipole emitter, is usually not possible for two reasons: (a) emitters in the near-field may couple to dark modes that are not excitable from the far-field and (b) spectral shifts appear between these two excitation schemes [41]. Nevertheless, the results from the far-field may be used as a rule of thumb to employ special characteristics of nanoantennas. In the case of the generalized Kerker condition, it would be desirable to use the directivity of nanoring antennas for close-by emitters. However, in such situation not just the directionality is important but also the Purcell factor and the antenna efficiency. In Fig. 5, the results for a dipole excitation of the nanoring is shown. The dipole is placed central to the bottom of the nanoring [Fig. 5 (a)]. The scattered field intensities in backand forward direction are displayed in Fig. 5 (a). They show that the scattering is mostly in the forward direction at $\nu_{\text {dip }} \approx 380 \mathrm{THz}$, see also the inset for the radiation pattern at $\nu_{\text {dip }}$. Note that a misplacement of the dipole by several tens of nanometers changes the radiation pattern of the antenna only slightly. The redshift of the spectral position of highest directivity can be explained by the difference between near- and farfield illumination [41]. The calculated emission rate enhancement, usually termed Purcell factor $F$, is calculated by the enhancement of the emitted power to the far-field compared to its emission without nanoantenna, $F=P_{\text {na }}^{\mathrm{rad}} / P_{\mathrm{fs}}^{\mathrm{rad}}[42]$. The antenna efficiency $\eta$ is calculated as the fraction of emitted power not dissipated by the nanoring to the total power emitted by the dipole [Fig. 5 (b)] [42]. Moderate Purcell factors around 12 are 
calculated for $\nu_{\text {dip }}$ with respect to efficiencies around 0.6. The efficiency dip around $\nu_{2} \approx 400 \mathrm{THz}$ can be attributed to the coupling to the quadrupolar mode of the nanoring. Hence, even though this coupling is necessary to achieve the high directivity, it leads to a comparably low efficiency for such large nanoantennas [43].

In conclusion we studied a novel condition to design highly directional plasmonic nanoantennas. The generalized Kerker condition relies on the constructive/destructive interference of different multipole moments in forward/backward direction. It has been verified for plasmonic nanorings in the case of plane-wave excitations and shows a predictive character for close-by dipoles as well. Our findings provide a powerful guideline to design highly directive plasmonic nanoantennas.

We thank the German Federal Ministry of Education and Research(PhoNa, 03IF2101A), the Thuringian State Government(MeMa, PE116-1) and the German Science Foundation (project RO 3640/3-1).

\section{References}

1. A. G. Curto, G. Volpe, T. H. Taminiau, M. P. Kreuzer, R. Quidant, and N. F. van Hulst, Science 329, 930 (2010).

2. A. E. Krasnok, C. R. Simovski, P. A. Belov, and Y. S. Kivshar, Nanoscale 6, 7354 (2014).

3. L. Novotny and N. van Hulst, Nat Photon 5, 83 (2011).

4. P. Bharadwaj, B. Deutsch, and L. Novotny, Adv. Opt. Photon. 1, 438 (2009).

5. R. Alaee, C. Menzel, U. Huebner, E. Pshenay-Severin, S. Bin Hasan, T. Pertsch, C. Rockstuhl, and F. Lederer, Nano Letters 13, 3482 (2013). PMID: 23805879.

6. S. Linden, C. Enkrich, M. Wegener, J. Zhou, T. Koschny, and C. M. Soukoulis, Science 306, 1351 (2004).

7. Z. Ruan and S. Fan, Phys. Rev. Lett. 105, 013901 (2010).

8. L. Verslegers, Z. Yu, Z. Ruan, P. B. Catrysse, and S. Fan, Phys. Rev. Lett. 108, 083902 (2012).

9. W. Cai, U. K. Chettiar, A. V. Kildishev, and V. M. Shalaev, Nat Photon 1, 224 (2007).

10. A. Alu and N. Engheta, Phys. Rev. Lett. 100, 113901 (2008).

11. S. S. Kruk, M. Decker, I. Staude, S. Schlecht, M. Greppmair, D. N. Neshev, and Y. S. Kivshar, ACS Photonics 1, 1218 (2014).

12. R. K. Rodriguez, S. F. B. Arango, P. Steinbusch, T. A. Verschuuren, M. F. Koenderink, A. and J. G. Rivas, Phys. Rev. Lett. 113, 247401 (2014).

13. S. Person, M. Jain, Z. Lapin, J. J. Saenz, G. Wicks, and L. Novotny, Nano Letters 13, 1806 (2013). PMID: 23461654 .

14. I. Staude, A. E. Miroshnichenko, M. Decker, N. T. Fofang, S. Liu, E. Gonzales, J. Dominguez, T. S. Luk, D. N. Neshev, I. Brener, and Y. Kivshar, ACS Nano 7, 7824 (2013).

15. M. Nieto-Vesperinas, R. Gomez-Medina, and J. J. Saenz, J. Opt. Soc. Am. A 28, 54 (2011).

16. T. Coenen, F. Bernal Arango, A. Femius Koenderink, and A. Polman, Nat Commun 5, 3250 (2014).

17. Y. H. Fu, A. I. Kuznetsov, A. E. Miroshnichenko, Y. F. Yu, and B. Luk/'yanchuk, Nat Commun 4, 1527 (2013).
18. T. Pakizeh and M. Kall, Nano Letters 9, 2343 (2009).

19. E. Rusak, I. Staude, M. Decker, J. Sautter, A. E. Miroshnichenko, D. A. Powell, D. N. Neshev, and Y. S. Kivshar, Applied Physics Letters 105, 221109 (2014).

20. D. Dregely, K. Lindfors, M. Lippitz, N. Engheta, M. Totzeck, and H. Giessen, Nat Commun 5, 4354 (2014).

21. M. Kerker, D.-S. Wang, and C. L. Giles, J. Opt. Soc. Am. 73, 765 (1983).

22. R. Alaee, M. Albooyeh, M. Yazdi, N. Komjani, C. Simovski, F. Lederer, and C. Rockstuhl, Phys. Rev. B 91, 115119 (2015).

23. X. Zambrana-Puyalto, I. Fernandez-Corbaton, M. L. Juan, X. Vidal, and G. Molina-Terriza, Opt. Lett. 38, 1857 (2013).

24. B. Rolly, B. Stout, and N. Bonod, Opt. Express 20, 20376 (2012).

25. I. M. Hancu, A. G. Curto, M. Castro-López, M. Kuttge, and N. F. van Hulst, Nano Letters 14, 166 (2013).

26. W. Liu, J. Zhang, B. Lei, H. Ma, W. Xie, and H. Hu, Opt. Express 22, 16178 (2014).

27. B. Rolly, J.-M. Geffrin, R. Abdeddaim, B. Stout, and N. Bonod, Sci. Rep. 3, 3063 (2013).

28. J. Aizpurua, P. Hanarp, D. S. Sutherland, M. Käll, G. W. Bryant, and F. J. Garcia de Abajo, Phys. Rev. Lett. 90, 057401 (2003).

29. F. Hao, P. Nordlander, Y. Sonnefraud, P. V. Dorpe, and S. A. Maier, ACS Nano 3, 643 (2009).

30. P. Nordlander, ACS Nano 3, 488 (2009).

31. C.-Y. Tsai, S.-P. Lu, J.-W. Lin, and P.-T. Lee, Applied Physics Letters 98, 153108 (2011).

32. Y. Sonnefraud, N. Verellen, H. Sobhani, G. A. Vandenbosch, V. V. Moshchalkov, P. Van Dorpe, P. Nordlander, and S. A. Maier, ACS Nano 4, 1664 (2010). PMID: 20155967.

33. D. Lehr, J. Reinhold, I. Thiele, H. Hartung, K. Dietrich, C. Menzel, T. Pertsch, E.-B. Kley, and A. Tünnermann, Nano Letters 15, 1025 (2015). PMID: 25584636.

34. A. Rakovich, P. Albella, and S. A. Maier, ACS Nano 0, null (0). PMID: 25602764.

35. J. D. Jackson, Classical Electrodynamics (Wiley, 1998), 3rd ed.

36. S. Mühlig, C. Menzel, C. Rockstuhl, and F. Lederer, Metamaterials 5, 64 (2011).

37. R. Gordon, Phys. Rev. B 73, 153405 (2006).

38. R. Filter, J. Qi, C. Rockstuhl, and F. Lederer, Phys. Rev. B 85, 125429 (2012).

39. D. Lehr, R. Alaee, R. Filter, K. Dietrich, T. Siefke, C. Rockstuhl, F. Lederer, E.-B. Kley, and A. Tünnermann, Applied Physics Letters 105 143110 (2014).

40. D. Lehr, K. Dietrich, C. Helgert, T. Käsebier, H.-J. Fuchs, A. Tünnermann, and E.-B. Kley, Opt. Lett. 37, 157 (2012).

41. P. Alonso-Gonzalez, P. Albella, F. Neubrech, C. Huck, J. Chen, F. Golmar, F. Casanova, L. E. Hueso, A. Pucci, J. Aizpurua, and R. Hillenbrand, Phys. Rev. Lett. 110, 203902 (2013).

42. R. Filter, S. Mühlig, T. Eichelkraut, C. Rockstuhl, and F. Lederer, Phys. Rev. B 86, 035404 (2012).

43. R. Filter, K. Słowik, J. Straubel, F. Lederer, and C. Rockstuhl, Opt. Lett. 39, 1246 (2014). 